BMJ Open

Diabetes

Research

\& Care

\title{
Association of smoking cessation after new-onset type 2 diabetes with overall and cause-specific mortality among Korean men: a nationwide population- based cohort study
}

\author{
Jae Woo Choi, ${ }^{1}$ Euna Han (D) , ${ }^{1}$ Tae Hyun Kim (D) ${ }^{2}$
}

To cite: Choi JW, Han E, Kim TH. Association of smoking cessation after new-onset type 2 diabetes with overall and cause-specific mortality among Korean men: a nationwide population-based cohort study. BMJ Open Diab Res Care 2020;8:e001249. doi:10.1136/ bmjdrc-2020-001249

\section{- Additional material is} published online only. To view please visit the journal online (http://dx.doi.org/10.1136/ bmjdrc-2020-001249).

Received 3 February 2020 Revised 8 May 2020 Accepted 2 June 2020

\section{Check for updates}

\section{(C) Author(s) (or their} employer(s)) 2020. Re-use permitted under CC BY-NC. No commercial re-use. See rights and permissions. Published by BMJ.

${ }^{1}$ College of Pharmacy, Yonsei University, Incheon, South Korea

${ }^{2}$ Graduate School of Public Health, Yonsei University, Seoul, South Korea

Correspondence to Dr Tae Hyun Kim; thkim@yuhs.ac

\section{ABSTRACT}

Introduction This study aimed to examine the association between smoking cessation after new-onset type 2 diabetes and overall and cause-specific mortality risks among Korean men.

Research design and methods The Korean National Health Insurance Service-National Health Screening Cohort database was searched, and 13377 Korean men aged $\geq 40$ years diagnosed with new-onset type 2 diabetes between 2004 and 2007 were included and followed up until 2013. We defined smoking status changes by comparing participants' answers in the last survey before diagnosis to those in the first survey after diagnosis. We estimated the adjusted HR (AHR) and 95\% Cl for mortality risk using multivariable Cox proportional hazards regression models. Results We identified 1014 all-cause mortality events (cancer, $n=406$ and cardiovascular disease (CVD), $n=184$ ) during an average follow-up duration of 7.2 years. After adjustment for all confounding factors, the reduced risk of all-cause mortality was more significant among short-term quitters (AHR 0.78; 95\% Cl 0.64 to 0.95), long-term quitters (AHR 0.68; $95 \% \mathrm{Cl} 0.54$ to 0.85 ), and never smokers (AHR 0.66; $95 \% \mathrm{Cl} 0.56$ to 0.78 ) compared with current smokers ( $p$ for trend $<0.001$ ). The lower risk of mortality from cancer was significant among the short-term quitters (AHR 0.60; 95\% Cl 0.44 to 0.83), long-term quitters (AHR $0.67 ; 95 \% \mathrm{Cl} 0.46$ to 0.90 ), and never smokers (AHR 0.50; $95 \% \mathrm{Cl} 0.39$ to 0.65 ) compared with current smokers ( $p$ for trend $<0.001$ ). There was no significant association between changes in smoking status and death from CVD. Smoking cessation after diagnosis in non-obese individuals (AHR $0.73 ; 95 \% \mathrm{Cl} 0.58$ to 0.92) and exercisers (AHR 0.54; 95\% Cl 0.38 to 0.76 ) was significantly associated with reduced mortality risk than current smoking.

Conclusions Smoking cessation after new-onset type 2 diabetes was associated with reduced mortality risk.

\section{INTRODUCTION}

Individuals with type 2 diabetes are at a higher mortality risk than those without diabetes. ${ }^{1}$ Smoking is a leading cause of premature mortality in patients with diabetes and the general population. ${ }^{2}$ A previous study

\section{Significance of this study}

What is already known about this subject?

- Although previous studies have demonstrated that quitting smoking decreases the risk of all-cause mortality in patients with diabetes, the effect of smoking cessation after new-onset type 2 diabetes on all-cause and cause-specific mortality compared with current smoking has not been assessed.

- There is limited evidence on the association between smoking cessation and mortality stratified by body mass index and exercise.

What are the new findings?

- Smoking cessation after new-onset type 2 diabetes was significantly associated with reduced mortality risk compared with current smoking.

> Smoking cessation after new-onset type 2 diabetes was associated with a reduced risk of mortality from cancer compared with current smoking.

- Smoking cessation after new-onset type 2 diabetes in non-obese and exerciser groups was associated with reduced mortality risk compared with current smoking.

How might these results change the focus of research or clinical practice?

- This study may provide additional evidence for clinicians for advising patients with type 2 diabetes to quit smoking.

involving women with type 2 diabetes has shown that current smokers have an increased mortality risk compared with never smokers. ${ }^{3}$ A meta-analysis has revealed that smoking amplifies the risk of mortality and cardiovascular events in patients with diabetes. ${ }^{4}$ Another meta-analysis has also indicated that active smoking is associated with significantly increased risks of total mortality and cardiovascular events compared with never smoking patients with diabetes. ${ }^{5}$ 


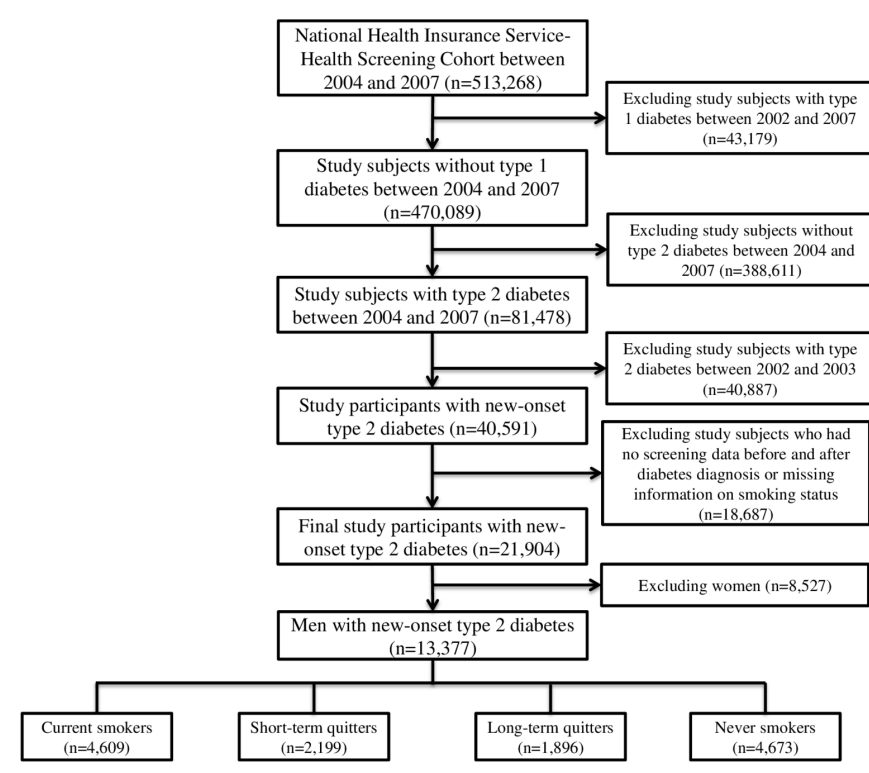

Figure 1 Flow chart of the study participants.

Although the negative health effects of smoking last long, smoking cessation appears to reduce the risk of adverse health outcomes on a long-term basis. ${ }^{67}$ However, the potential reduction in mortality risk by quitting smoking is not clear in patients with type 2 diabetes. A prior study has examined the effects of quitting smoking on mortality risk in patients with diabetes. ${ }^{8}$ Another study has assessed the association of quitting smoking and mortality in women with type 2 diabetes. ${ }^{3}$ A recent study has examined the effects of quitting smoking and body mass index (BMI) changes on all-cause mortality in patients with diabetes. ${ }^{9}$ However, the effects of smoking cessation after new-onset type 2 diabetes on overall and cause-specific mortality have not been investigated.

Smoking cessation is a major target for diabetes treatment. ${ }^{10}$ However, many patients continue to smoke even after being diagnosed with diabetes mellitus, ${ }^{11}$ and the success rate of smoking cessation interventions among patients with diabetes is low (approximately 20\%). ${ }^{12}$ This study may provide additional evidence for the likely benefits of quitting smoking after the diagnosis of type 2 diabetes.

We examined the potential benefits of smoking cessation after new-onset type 2 diabetes in terms of reducing the risk of all-cause mortality using nationwide retrospective cohort data. We also explored the association between smoking cessation and deaths from cancer and cardiovascular disease (CVD) because cancer and CVD are the major causes of worldwide deaths,${ }^{13}$ for which smoking is an important risk factor. ${ }^{14}{ }^{15}$ Further, we identified the relationship between smoking cessation and allcause mortality stratified by BMI and exercise.

\section{MATERIALS AND METHODS}

\section{Data and study sample}

The study data were extracted from the Korean National Health Insurance Service (NHIS)-National Health
Screening Cohort (HEALS) database between 2002 and 2013. ${ }^{16}$ The Korean NHIS operates a mandatory health insurance system and covers approximately $97 \%$ of the Korean population. All subscribers aged 40-79 years are required to have a standardized biannual health screening (annual for manual workers). The NHISHEALS was extracted using a simple random sampling method to establish a representative sample and comprised 10\% (514 866 individuals) of all health examination participants from 2002 to 2003 who were followed up until 2013. The NHIS-HEALS data include demographics and clinical information regarding diagnoses, treatments, and prescribed drugs for all visits (inpatient, outpatient, and pharmacy visits) to healthcare facilities by cohort subjects. The NHIS-HEALS data also include national health screening information, performed biennially from 2002 to 2013, consisting of chest radiographic examinations, regular blood tests, a survey questionnaire on medical history, and physical examinations.

Among the 513268 participants from the NHIS-HEALS between 2004 and 2007, we excluded study subjects with type 1 diabetes between 2002 and $2007(n=43179)$. We also excluded subjects without type 2 diabetes to solely recruit patients with type 2 diabetes as study participants between 2004 and 2007 ( $\mathrm{n}=388611)$. Type 2 diabetes was defined as the existence of any one of the following criteria: (1) fasting blood glucose level of $\geq 7 \mathrm{mmol} / \mathrm{L}$ (126 mg/dL); (2) physician's diagnosis of type 2 diabetes (the International Classification of Diseases 10th Revision (ICD-10) code: E11-E14) more than twice within 6 months; (3) treatment with an oral hyperglycemic medication or insulin (online supplementary table 1). We also excluded subjects with type 2 diabetes between 2002 and 2003 to include only patients with new-onset type 2 diabetes $(n=40887)$. Finally, we excluded subjects who had no screening data before and after the diagnosis of diabetes or had missing information on smoking status $(\mathrm{n}=18687)$ and excluded women $(\mathrm{n}=8527)$ because there was a significantly low proportion of current women smokers at baseline (below 5\%). Finally, 13377 subjects with newly diagnosed type 2 diabetes were included (figure 1).

\section{Measurement}

The dependent variables in this study were all-cause and cause-specific mortality. The cohort database was linked to the death registration database of Statistics Korea, which included the causes and dates of mortality. The various causes of death were coded according to ICD-10. Cause-specific mortality was classified as deaths from cancer (ICD-10 C00-C99) and CVD (ICD-10 I00-I99) based on previous studies. ${ }^{17}$ The survival duration was measured in days, and all subjects were followed up until mortality, withdrawal from the medical security system, or the end of 2013, whichever occurred first. The percentage of cases in which the status was not available at the end of the follow-up was approximately $0.5 \%$. 
We used the smoking status assessed from the selfreported questionnaire surveyed in the national health screening as the independent variable. The questionnaire items regarding the smoking status included non-smoking, ex-smoking, and current smoking. We compared the participants' answers on the smoking status in the latest survey before the diagnosis of diabetes with those in the first survey conducted after the diagnosis; the study subjects were divided into four groups based on the changes in their smoking status-current smoker, shortterm quitter, long-term quitter, and never smoker. The study participants were classified as current smokers if they answered current smoking after diagnosis regardless of smoking status before diagnosis $(n=3857)$; short-term quitters if they answered: (1) current smoking before diagnosis and non-smoking after diagnosis $(n=942)$, (2) current smoking before diagnosis and ex-smoking after diagnosis $(n=489)$, or (3) non-smoking before diagnosis and ex-smoking after diagnosis $(\mathrm{n}=768)$; long-term quitters if they answered: (1) ex-smoking both before and after diagnosis $(\mathrm{n}=859)$ or (2) ex-smoking before diagnosis and non-smoking after diagnosis $(n=1037)$; and never smokers if they answered non-smoking both before and after diagnosis $(n=4673)$. The surveyed date of smoking status after the diagnosis was defined as index date (start date of follow-up) in our study.

The potential confounding factors considered in analyses available for adjustment were age, residential area, household income, BMI, systolic and diastolic blood pressures (BP), fasting blood glucose level, total cholesterol level, family history of diabetes, heavy drinking, exercise, and comorbidities. All potential confounding factors, except comorbidities, were measured at the index date.

Household income was classified as low $(<40$ th percentile), middle (41st-80th percentiles), or high (81st-100th percentiles). Residential area was classified as metropolitan (capital), urban (local government where $>1$ million individuals live), or rural (other). The WHO recommendations for Asian populations were used to categorize the individuals into five BMI groups: $<18.5 \mathrm{~kg} / \mathrm{m}^{2}$ (underweight), $18.5-22.9 \mathrm{~kg} / \mathrm{m}^{2}$ (normal), $23.0-24.9 \mathrm{~kg} / \mathrm{m}^{2}$ (overweight), $25.0-29.9 \mathrm{~kg} / \mathrm{m}^{2}$ (class I obese), or $\geq 30 \mathrm{~kg}$ / $\mathrm{m}^{2}$ (class II obese). ${ }^{18}$ Individuals who consumed $\geq 30 \mathrm{~g} /$ day of alcohol were defined as heavy drinkers, ${ }^{19}$ those who drank alcohol but not heavily were defined as nonheavy drinkers, and those who did not drink alcohol were defined as non-drinkers. Exercise was defined as performance of exercise at least once a week. Systolic and diastolic BPs were measured with subjects seated and after at least $5 \mathrm{~min}$ of rest. Blood samples were obtained after overnight fasting to measure the serum glucose, total cholesterol, and creatinine levels.

The comorbidities consisted of hypertension (ICD-10: I10-I15), renal failure (ICD-10: N17-N19), stroke (ICD10: I60-I63), ischemic heart disease (ICD-10: I20-I25), and cancer (ICD-10: C00-C99) and were assessed by screening information for physician's diagnoses of study subjects before index date.

\section{Statistical analyses}

The clinical and demographic characteristics of the study participants were compared based on the change in their smoking status using analysis of variance for continuous variables and Pearson's $\chi^{2}$ test for categorical variables. Data were presented as mean \pm SD for continuous variables and as numbers (percentages) for categorical variables. For each participant, mortality was measured from the index date to 31 December 2013. The number of personyears of follow-up was counted and age-standardized mortality rates were estimated from changes in smoking status (online supplementary table 2).

Cox proportional hazards regression models were used to evaluate the association between smoking cessation and mortality, and adjusted HR (AHR) and 95\% CI were calculated using the potential confounding factors described in table 1 . The functional form of continuous variables (age, systolic BP, diastolic BP, fasting glucose level, and total cholesterol level) was assessed and the models were stratified by non-proportional covariates (systolic BP, diastolic BP, and total cholesterol level) (online supplementary figure 1). First, we analyzed the effect of smoking cessation after new-onset type 2 diabetes on all-cause mortality. Second, we identified the association between smoking cessation and mortality from cancer and CVD. Finally, we examined the relationship between smoking cessation and mortality stratified by BMI and exercise.

All data extraction and statistical analyses were performed using Statistical Analysis System (SAS) V.9.4 (SAS Institute). Proportional hazards assumptions were evaluated statistically and satisfied for all models.

\section{RESULTS}

Our study enrolled 13377 individuals aged $\geq 40$ years with incident type 2 diabetes. We identified 1014 events of allcause mortality (cancer, $n=406$ and CVD, $n=184$ ) during an average follow-up duration of $7.2 \pm 1.4$ years. Table 1 presents the general characteristics of study subjects based on changes in the smoking status. The proportions of subjects with obesity, high household income, and comorbidities were higher among short-term quitters than among current smokers. In contrast, the proportions of subjects living in urban residential areas, with a family history of diabetes, who drank heavily, and who exercised were lower among the short-term quitters than among current smokers.

Figure 2 presents the AHRs and 95\% CIs for the risk of overall and cause-specific mortality based on changes in smoking status (online supplementary table 2). After adjustment for all confounding factors, the reduced risk of all-cause mortality was more significant among shortterm quitters (AHR $0.78 ; 95 \%$ CI 0.64 to 0.95 ), long-term quitters (AHR 0.68; 95\% CI 0.54 to 0.85 ), and never smokers (AHR 0.66; 95\% CI 0.56 to 0.78 ) compared with current smokers ( $\mathrm{p}$ for trend $<0.001$ ). The lower risk of mortality from cancer was significant among the 
Table 1 General characteristics of study subjects according to change of smoking status

\begin{tabular}{|c|c|c|c|c|c|c|c|c|c|c|}
\hline \multirow[b]{3}{*}{ Variables } & \multirow[b]{3}{*}{ Total } & \multicolumn{8}{|c|}{ Change of smoking status } & \multirow[b]{3}{*}{$P$ value } \\
\hline & & \multicolumn{2}{|c|}{$\begin{array}{l}\text { Current } \\
\text { smokers }\end{array}$} & \multicolumn{2}{|c|}{$\begin{array}{l}\text { Short-term } \\
\text { quitters }\end{array}$} & \multicolumn{2}{|c|}{$\begin{array}{l}\text { Long-term } \\
\text { quitters }\end{array}$} & \multicolumn{2}{|c|}{ Never smokers } & \\
\hline & & $\mathbf{n}$ & $\%$ & $\mathbf{n}$ & $\%$ & $\mathbf{n}$ & $\%$ & $\mathbf{n}$ & $\%$ & \\
\hline Total & 13377 & 4609 & 34.5 & 2199 & 16.4 & 1896 & 14.2 & 4673 & 34.9 & \\
\hline Age (years) & $56.4 \pm 9.1$ & 54.4 & 8.5 & 57.0 & 9.0 & 56.6 & 9.3 & 58.1 & 9.3 & $<0.001$ \\
\hline BMI $\left(\mathrm{kg} / \mathrm{m}^{2}\right)$ & & & & & & & & & & $<0.001$ \\
\hline$\leq 18.5$ & 244 & 130 & 2.8 & 24 & 1.1 & 24 & 1.3 & 66 & 1.4 & \\
\hline $18.5-23$ & 3842 & 1523 & 33.0 & 588 & 26.7 & 464 & 24.5 & 1267 & 27.1 & \\
\hline $23-25$ & 3781 & 1263 & 27.4 & 611 & 27.8 & 558 & 29.4 & 1349 & 28.9 & \\
\hline $25-30$ & 5085 & 1548 & 33.6 & 906 & 41.2 & 783 & 41.3 & 1848 & 39.5 & \\
\hline$\geq 30$ & 425 & 145 & 3.1 & 70 & 3.2 & 67 & 3.5 & 143 & 3.1 & \\
\hline \multicolumn{11}{|l|}{$\mathrm{BP}(\mathrm{mm} \mathrm{Hg})$} \\
\hline Systolic & $130.1 \pm 16.1$ & 129.1 & 16.0 & 130.0 & 16.1 & 130.4 & 15.3 & 131.0 & 16.5 & $<0.001$ \\
\hline Diastolic & $81.2 \pm 10.5$ & 80.8 & 10.5 & 81.0 & 10.6 & 81.5 & 10.2 & 81.4 & 10.6 & 0.016 \\
\hline Fasting glucose (mg/dL) & $111.0 \pm 33.6$ & 111.2 & 35.3 & 111.7 & 38.1 & 110.7 & 29.4 & 110.6 & 31.2 & 0.544 \\
\hline Total cholesterol (mg/dL) & $196.3 \pm 37.5$ & 197.4 & 37.6 & 197.2 & 38.3 & 197.0 & 36.4 & 194.6 & 37.5 & 0.001 \\
\hline Family history of diabetes & 1014 & 372 & 8.1 & 158 & 7.2 & 168 & 8.9 & 316 & 6.8 & 0.014 \\
\hline Heavy drinking & 1121 & 588 & 12.8 & 164 & 7.5 & 136 & 7.2 & 233 & 5.0 & $<0.001$ \\
\hline Exercise & 7547 & 2574 & 55.8 & 1157 & 52.6 & 1198 & 63.2 & 2618 & 56.0 & $<0.001$ \\
\hline Household income & & & & & & & & & & $<0.001$ \\
\hline Low & 3598 & 1354 & 29.4 & 572 & 26.0 & 399 & 21.0 & 1273 & 27.2 & \\
\hline Middle & 5173 & 1883 & 40.9 & 856 & 38.9 & 699 & 36.9 & 1735 & 37.1 & \\
\hline High & 4606 & 1372 & 29.8 & 771 & 35.1 & 798 & 42.1 & 1665 & 35.6 & \\
\hline Residential area & & & & & & & & & & $<0.001$ \\
\hline Metropolitan & 2068 & 619 & 13.4 & 362 & 16.5 & 320 & 16.9 & 767 & 16.4 & \\
\hline Urban & 3963 & 1481 & 32.1 & 596 & 27.1 & 541 & 28.5 & 1345 & 28.8 & \\
\hline Rural & 7346 & 2509 & 54.4 & 1241 & 56.4 & 1035 & 54.6 & 2561 & 54.8 & \\
\hline \multicolumn{11}{|l|}{ Comorbidities } \\
\hline Hypertension & 6032 & 1720 & 37.3 & 1038 & 47.2 & 915 & 48.3 & 2359 & 50.5 & $<0.001$ \\
\hline Renal failure & 198 & 46 & 1.0 & 40 & 1.8 & 32 & 1.7 & 80 & 1.7 & 0.010 \\
\hline Stroke & 636 & 158 & 3.4 & 104 & 4.7 & 107 & 5.6 & 267 & 5.7 & $<0.001$ \\
\hline Ischemic heart disease & 2019 & 561 & 12.2 & 352 & 16.0 & 335 & 17.7 & 771 & 16.5 & $<0.001$ \\
\hline Cancer & 1060 & 284 & 6.2 & 200 & 9.1 & 162 & 8.5 & 414 & 8.9 & $<0.001$ \\
\hline
\end{tabular}

Values are presented as mean \pm SD or $\mathrm{n}(\%)$.

$\mathrm{BMI}$, body mass index; BP, blood pressure.

short-term quitters (AHR 0.60; 95\% CI 0.44 to 0.83 ), long-term quitters (AHR 0.67; 95\% CI 0.46 to 0.90 ), and never smokers (AHR 0.50; $95 \%$ CI 0.39 to 0.65 ) compared with current smokers ( $\mathrm{p}$ for trend $<0.001$ ). There was no significant association between changes in smoking status and death from CVD.

Figure 3 shows the AHRs and 95\% CIs for the risk of all-cause mortality for individuals with different smoking behaviors stratified by BMI and exercise (online supplementary table 3). In the non-obese (individuals with BMI $<25 \mathrm{~kg} / \mathrm{m}^{2}$ ) and exerciser groups, reduced risk of mortality was significant among short-term quitters (AHR 0.73, 0.54; $95 \%$ CI 0.58 to $0.92,95 \%$ CI 0.38 to 0.76 , respectively), long-term quitters (AHR 0.67, 0.58; 95\% CI 0.52 to 0.87 , $95 \%$ CI 0.42 to 0.80 , respectively), and never smokers (AHR 0.64, 0.58; $95 \%$ CI 0.53 to $0.78,95 \%$ CI 0.45 to 0.75 , respectively) compared with current smokers. Lower risk of mortality was observed among long-term quitters in obese group (individuals with BMI $>25 \mathrm{~kg} / \mathrm{m}^{2}$ ) (AHR 0.61; $95 \%$ CI 0.40 to 0.93 ) and never smokers in the obese (AHR $0.61 ; 95 \%$ CI 0.44 to 0.84 ) and exerciser groups (AHR $0.72 ; 95 \%$ CI 0.58 to 0.90 ) compared with current smokers.

\section{DISCUSSION}

This study had three main findings. First, smoking cessation after new-onset type 2 diabetes was associated with 


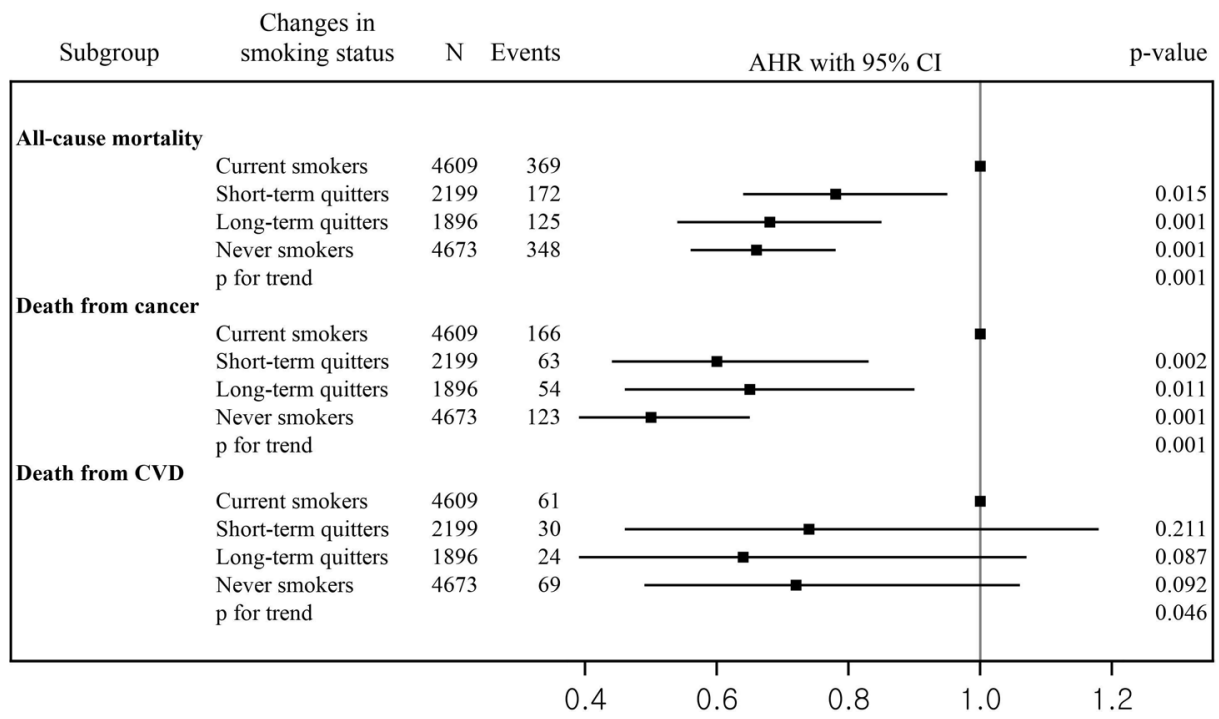

Figure 2 Risk of overall and cause-specific mortality based on change of smoking status. AHR, adjusted HR; CVD, cardiovascular disease.

reduced risk of all-cause mortality compared with current smoking. Second, smoking cessation after new-onset type 2 diabetes was significantly associated with a lower risk of mortality from cancer compared with current smoking. Third, smoking cessation after new-onset type 2 diabetes in the non-obese and exerciser groups was associated with a lower risk of mortality compared with current smoking.

The findings of previous studies regarding the effect of smoking status on mortality have been unclear. ${ }^{389}$ In a study by Chaturvedi $e t$ al, the all-cause mortality risks were higher for quitters than for never smokers. ${ }^{8}$ In the study by Al-Delaimy $e t a l$, women with type 2 diabetes who had stopped smoking for $\geq 10$ years had lower mortality risk than never smokers ${ }^{3}$; however, they used the never smoking group as a reference group and it is difficult to identify the benefits of smoking cessation over current smoking. Cho et al investigated the association between smoking cessation by BMI changes and mortality and used non-reducers who did not reduce their smoking amount among current smokers as a reference group. ${ }^{9}$ However, they did not observe any benefits of smoking cessation on mortality. Our study suggests benefits of

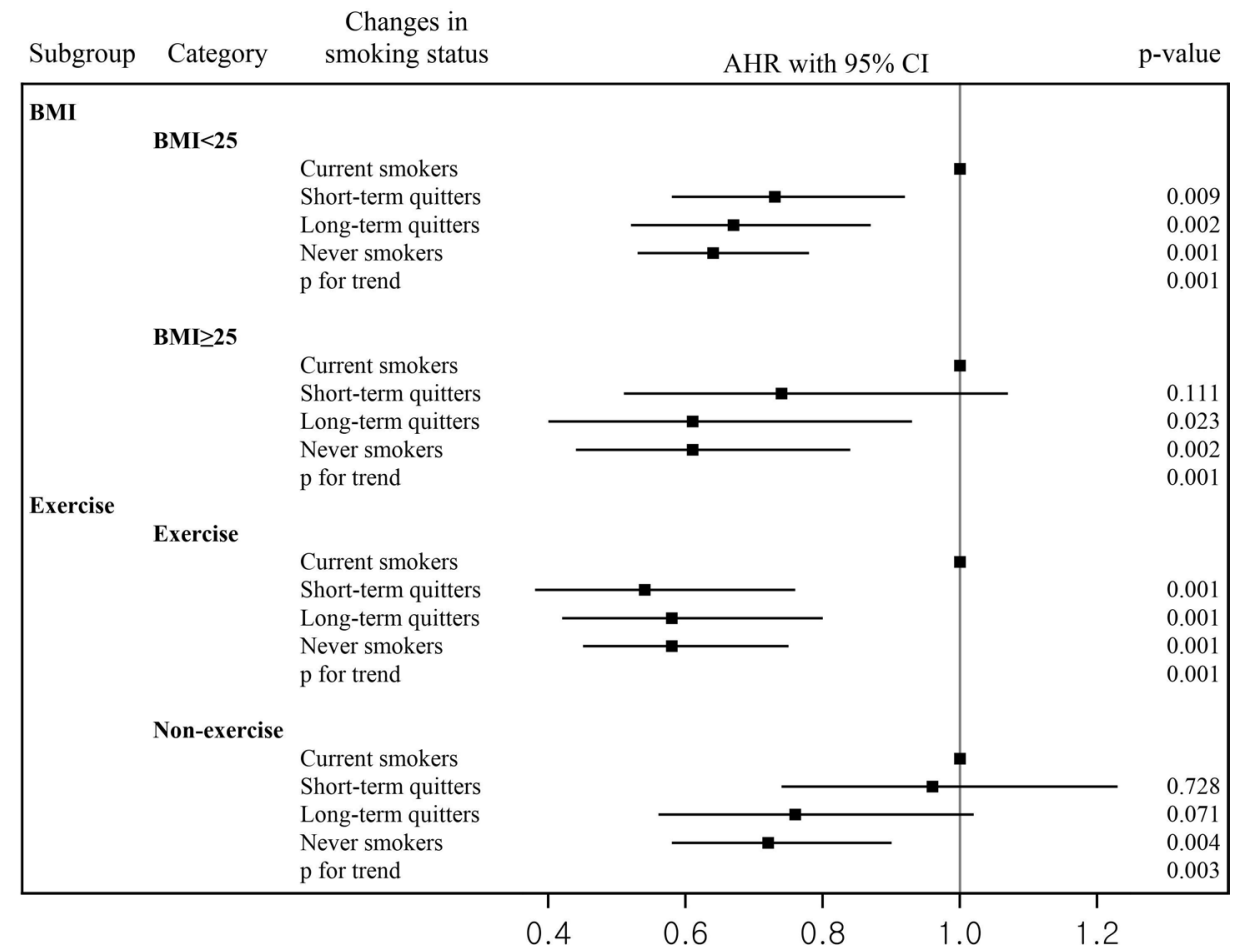

Figure 3 Change of smoking status and risk of mortality as stratified by BMI and exercise. AHR, adjusted HR; BMI, body mass index. 
smoking cessation on mortality. Smoking cessation is particularly important for patients with type 2 diabetes because their risk of developing other morbidities is substantially augmented by smoking and insulin resistance. ${ }^{20}$ In our study, smoking cessation after the diagnosis of type 2 diabetes was significantly associated with a reduced risk of all-cause mortality compared with current smoking. This indicates that quitting smoking may have health benefits after the diagnosis of type 2 diabetes.

We found that smoking cessation after new-onset type 2 diabetes is significantly associated with a lower risk of mortality from cancer compared with current smoking, which is consistent with previous reports. In a study by Ohnishi et $a l,{ }^{21}$ current smokers with type 2 diabetes had a higher risk of cancer mortality. ${ }^{21}$ Type 2 diabetes in itself is associated with cancer mortality. ${ }^{22}{ }^{23}$ Hyperinsulinemia accompanied by insulin resistance, chronic inflammation, and hyperglycemia are known mechanisms of oncogenesis. Another reason for the association of type 2 diabetes with cancer is that patients with type 2 diabetes are likely to have common cancer risk factors, such as smoking, obesity, and alcohol drinking. Cigarette smoking is a strong risk factor for various types of cancer, ${ }^{24}$ and therefore, smoking cessation is encouraged among patients with type 2 diabetes.

One of the main findings of this study is that smoking cessation after new-onset type 2 diabetes in non-obese and exerciser groups was associated with a reduced risk of mortality compared with current smoking. However, in the obese and non-exerciser groups, there were no associations between short-term smoking cessation after diagnosis of type 2 diabetes and mortality. Obesity or physical inactivity appears to counteract the survival benefits of smoking cessation. ${ }^{25}{ }^{26}$ Hence, the evidence suggests that the benefits of smoking cessation may only be valid for relatively healthier patients with type 2 diabetes.

This study had several limitations. First, the smoking status was assessed on the basis of patients' self-reported information without biochemical verification, which led to underestimation or overestimation of smoking cessation. We also could not determine the exact time of smoking cessation in the study subjects because of limited data availability. Further research is warranted to explore this association using data on the exact time of smoking cessation and biomarker test findings, such as urine cotinine levels. Second, although we attempted to adjust for diverse confounding variables using multivariate analyses, the possibility of residual confounding factors cannot be completely excluded. Third, the participation level in Korean national health screening was approximately $60 \%$ in 2007, and therefore, there may have been possible selection bias. Fourth, there may be possibilities for misdiagnosis of type 2 diabetes. Although type 2 diabetes can be diagnosed using HbAlc or oral glucose tolerance test in addition to the fasting blood glucose test, the information for the former two was not included in NHIS-HEALS data. The fasting blood glucose test results were based on a single measurement; however, the test usually needs to be repeated on a second day to diagnose diabetes. Furthermore, patients with type 3 diabetes may have been included as study subjects; we could not exclude them. Finally, our study included only a Korean population, and thus, the findings cannot be generalized to other ethnicities.

The strengths of our study include its longitudinal design and availability of abundant demographic, lifestyle variable, comorbidity, and biomedical data. To our knowledge, this is the first study to examine the impact of smoking cessation after new-onset type 2 diabetes on the risk of mortality.

In conclusion, this cohort study of a Korean population demonstrated that smoking cessation after new-onset type 2 diabetes was associated with reduced risk of allcause mortality. Although smoking cessation seems to be more effective before the diagnosis of diabetes, quitting smoking after the diagnosis of diabetes has health benefits. We suggest that clinicians should encourage smokers with type 2 diabetes to quit smoking.

Acknowledgements We thank Editage (www.editage.co.kr) for English language editing.

Contributors JWC, EH and THK designed the study. JWC and EH performed the literature review and interpretation for data analysis. JWC analyzed the data. JWC, EH and THK wrote the draft. All authors read and approved the final manuscript.

Funding This work was supported by the National Research Foundation of Korea (grant number: 2019R1A2C1003259), the Yonsei University Research Fund (Post Doc. Researcher Supporting Program) of 2019 (grant number: 2019-12-0129), and the National Research Foundation of Korea (grant number: 2020R1I1A1A01053104).

\section{Competing interests None declared.}

\section{Patient consent for publication Not required.}

Ethics approval The Yonsei University Institutional Review Board approved this study (approval number: 7001988-202004-HR-818-02E) and the requirement for informed consent was waived as the NHIS-HEALS database was constructed after anonymization according to strict confidentiality guidelines.

Provenance and peer review Not commissioned; externally peer reviewed.

Data availability statement Data may be obtained from a third party and are not publicly available.

Open access This is an open access article distributed in accordance with the Creative Commons Attribution Non Commercial (CC BY-NC 4.0) license, which permits others to distribute, remix, adapt, build upon this work non-commercially, and license their derivative works on different terms, provided the original work is properly cited, appropriate credit is given, any changes made indicated, and the use is non-commercial. See: http://creativecommons.org/licenses/by-nc/4.0/.

ORCID iDs

Euna Han http://orcid.org/0000-0003-2656-7059

Tae Hyun Kim http://orcid.org/0000-0003-1053-8958

\section{REFERENCES}

1 Yang JJ, Yu D, Wen W, et al. Association of diabetes with all-cause and cause-specific mortality in Asia: a pooled analysis of more than 1 million participants. JAMA Netw Open 2019;2:e192696.

2 Fielding JE. Smoking: health effects and control (1). N Engl J Med 1985;313:491-8.

3 Al-Delaimy WK, Willett WC, Manson JE, et al. Smoking and mortality among women with type 2 diabetes: the nurses' health study cohort. Diabetes Care 2001;24:2043-8.

4 Qin R, Chen T, Lou Q, et al. Excess risk of mortality and cardiovascular events associated with smoking among patients with diabetes: meta-analysis of observational prospective studies. Int $J$ Cardiol 2013;167:342-50. 
5 Pan A, Wang Y, Talaei M, et al. Relation of smoking with total mortality and cardiovascular events among patients with diabetes mellitus: a meta-analysis and systematic review. Circulation 2015;132:1795-804.

6 Lightwood JM, Glantz SA. Short-Term economic and health benefits of smoking cessation: myocardial infarction and stroke. Circulation 1997;96:1089-96.

7 Scanlon PD, Connett JE, Waller LA, et al. Smoking cessation and lung function in mild-to-moderate chronic obstructive pulmonary disease. the lung health study. Am J Respir Crit Care Med 2000;161:381-90.

8 Chaturvedi N, Stevens L, Fuller JH. Which features of smoking determine mortality risk in former cigarette smokers with diabetes? Diabetes Care 1997;20:1266-72.

9 Cho MH, Lee K, Park SM, et al. Effects of smoking habit change on all-cause mortality and cardiovascular diseases among patients with newly diagnosed diabetes in Korea. Sci Rep 2018;8:5316.

10 American Diabetes Association. Standards of medical care in diabetes-2015 abridged for primary care providers. Clin Diabetes 2015;33:97-111.

11 Resnick HE, Foster GL, Bardsley J, et al. Achievement of American diabetes association clinical practice recommendations among U.S. adults with diabetes, 1999-2002: the National health and nutrition examination survey. Diabetes Care 2006;29:531-7.

12 Tonstad S. Cigarette smoking, smoking cessation, and diabetes. Diabetes Res Clin Pract 2009;85:4-13.

13 Wang XJ, Jiang CQ, Zhang WS, et al. Milk consumption and risk of mortality from all-cause, cardiovascular disease and cancer in older people. Clin Nutr 2020. doi:10.1016/..clnu.2020.03.003

14 Batty GD, Kivimaki M, Gray L, et al. Cigarette smoking and sitespecific cancer mortality: testing uncertain associations using extended follow-up of the original Whitehall study. Ann Oncol 2008;19:996-1002.

15 Ezzati M, Henley SJ, Thun MJ, et al. Role of smoking in global and regional cardiovascular mortality. Circulation 2005;112:489-97.
16 Seong SC, Kim Y-Y, Park SK, et al. Cohort profile: the National health insurance Service-National health screening cohort (NHIS-HEALS) in Korea. BMJ Open 2017;7:e016640.

17 Liu Y, Shu X-O, Wen W, et al. Association of leisure-time physical activity with total and cause-specific mortality: a pooled analysis of nearly a half million adults in the Asia cohort Consortium. Int $J$ Epidemiol 2018;47:771-9.

18 WHO Expert Consultation. Appropriate body-mass index for Asian populations and its implications for policy and intervention strategies. Lancet 2004;363:157-63.

19 Agarwal DP. Cardioprotective effects of light-moderate consumption of alcohol: a review of putative mechanisms. Alcohol Alcohol 2002;37:409-15.

20 Al-Delaimy WK, Manson JE, Solomon CG, et al. Smoking and risk of coronary heart disease among women with type 2 diabetes mellitus. Arch Intern Med 2002;162:273-9.

21 Ohnishi H, Saitoh S, Akasaka H, et al. Combination of type 2 diabetes and smoking increases total cancer mortality in Japanese men using competing risk analysis: the Tanno-Sobetsu study. Diabetol Int 2016;7:167-72.

22 Sasazuki S, Charvat H, Hara A, et al. Research Group for the D, evaluation of cancer prevention strategies in J. diabetes mellitus and cancer risk: pooled analysis of eight cohort studies in Japan. Cancer Sci 2013;104:1499-507.

23 Noto H, Tsujimoto T, Sasazuki T, et al. Significantly increased risk of cancer in patients with diabetes mellitus: a systematic review and meta-analysis. Endocr Pract 2011;17:616-28.

24 IARC Working Group on the Evaluation of Carcinogenic Risks to Humans. Tobacco smoke and involuntary smoking. IARC Monogr Eval Carcinog Risks Hum 2004;83:1-1438.

25 Shaw BA, Agahi N. Smoking and physical inactivity patterns during midlife as predictors of all-cause mortality and disability: a 39-year prospective study. Eur J Ageing 2014;11:195-204.

26 Luijckx E, Lohse T, Faeh D, et al. Joints effects of BMI and smoking on mortality of all-causes, CVD, and cancer. Cancer Causes Control 2019;30:549-57. 